\title{
Accesibilidad al patrimonio cultural para débiles visuales y ciegos en el Centro Histórico de Puebla. Una experiencia táctil
}

Cultural heritage accessibility for persons with visual
weak and blinds in the Historic Center of Puebla.
Tactile experience

DOI: 10.17981/mod.arq.cuc.18.2.2018.07

Fecha de Envío: 13/09/2018 Fecha de Aceptación: 11/09/2018

\section{Adriana Hernández Sánchez ${ }^{1}$,}

\section{Christian Enrique de la Torre Sánchez ${ }^{2}$ y Francisco Javier Vázquez ${ }^{3}$}

Benemérita Universidad Autónoma de Puebla - BUAP (México) adna0909@hotmail.com

Para citar este artículo:

Hernández, A., De la Torre, C. y Vásquez, J. (2018). Accesibilidad al patrimonio cultural para débiles visuales y ciegos en el Centro Histórico de Puebla. Una experiencia táctil. MODULO ARQUITECTURA-CUC, vol. 21, no. 1, pp. 181-199. DOI: 10.17981/mod.arq.cuc.18.2.2018.07

\section{Resumen}

En términos generales, el patrimonio carece de una estrategia de accesibilidad, que se acentúa en aquellas personas que presentan ceguera y debilidad visual. Por lo cual, es necesario ofrecer alternativas para que el patrimonio pueda ser conocido o reconocido, por cualquier persona. En sus diferentes etapas, el proyecto de investigación desarrollado por la facultad de Arquitectura de la Benemérita Universidad Autónoma de Puebla y Re Genera Espacio, plantea estrategias de accesibilidad hacia el patrimonio desarrollando una maqueta en $3 \mathrm{D}$, de espacios arquitectónicos poco difundidos, obteniendo una mejor lectura del patrimonio por parte de los usuarios a través de la volumetría y el reconocimiento a detalle de los elementos. En este proceso, el equipo de trabajo se involucró con una asociación de ciegos y débiles visuales, experimentando sobre el tipo de materiales, colores y dimensiones.

Palabras clave: Accesibilidad, maquetas, ciegos, débil visual, táctil.

\section{Abstract}

Heritage conservation does not have an strategy of accessibility for blind people and persons with visual weakness. It is necessary to offer alternatives so that the patrimony can be known, or recognized, by any person. In its different stages, the research project developed by the faculty of Architecture - Benemérita Universidad Autónoma (Puebla) and Re Genera Espacio, raises strategies of accessibility to heritage by developing a 3D model of little-known architectural spaces, obtaining a better reading of the heritage by the users through the volumetry and the detailed recognition of the elements. In this process, the work team was involved with an association of blinds and visually impaireds persons, experimenting on the type of materials, colors and dimensions.

Keywords: Accessibility, models, blind, visual weakness, tactile.

1 Doctora en Espacio Público, Regeneración Urbana: Arte, Teoría y Conservación del Patrimonio por la Universitat de Barcelona Maestra en Diseño Arquitectónico por la UNAM y Arquitecta por la BUAP, perteneciente a la línea de Investigación "Espacio Público, Participación Ciudadana y Centro Histórico" de la Benemérita Universidad Autónoma de Puebla, Puebla (México).

2 Doctorante en Procesos Territoriales de la BUAP. Maestro en Conservación del patrimonio por la BUAP, Puebla (México).

3 Estudiante de licenciatura en Arquitectura de la BUAP y becario del programa Jóvenes Investigadores VIEP 2017, Puebla (México).

- The author; licensee Universidad de la Costa - CUC.

Módulo Arquitectura CUC vol. 21 no. 1, pp. 181-199. Julio - Diciembre, 2018

Barranquilla. ISSN Impreso 0124-6542, ISSN Online 2389-7732 


\section{INTRODUCCIÓN}

El patrimonio arquitectónico y artístico de las ciudades históricas es desconocido por la mayoría de las personas con discapacidad. Las personas ciegas y débiles visuales tienen opciones limitadas o inexistentes, debido a la existente oferta de difusión y museografía basada en la lectura y la apreciación visual. Por tales motivos, algunas entidades como la Red de Museos Locales de la Oficina de Patrimonio Cultural de la Diputación de Barcelona, en un proyecto denominado "La mirada táctil 2017-2024 una degustación", mencionan que la promoción de elementos museísticos táctiles tiene como finalidad ofrecer una primera experiencia sensorial de los contenidos de los recintos para llegar a conocer su esencia a partir del tacto, como parte de una oferta más amplia e incluyente dirigida al público en general. Mencionan además que la oferta de elementos táctiles en los museos comienza con lo denominado "intocables táctiles", es decir, aquellos elementos que, por legislación, seguridad, ubicación, antigüedad, deterioro, condiciones o simbolismo, no pueden ser tocados por cualquier persona; al incluir a los especialistas que requieren de elementos adicionales de protección como guantes, películas plásticas, papeles especiales o revisión con cámara para evitar el contacto con ellos. Al hablar de elementos artísticos y arquitectónicos inaccesibles para el público en general, no solo para ciegos y débiles visuales, se hace necesario para una mejor valoración del patrimonio de elementos presentados en formatos apreciables de manera táctil, como maquetas, planos y relieves. Hay extraordinarios referentes españoles y argentinos de estas técnicas, entre los cuales podemos mencionar la Casa de la Pedrera (Barcelona) y la Catedral de Valencia, además del discurso museográfico del MARQ (Alicante) y el Vila Museu (Villajoyosa), por el esfuerzo que realiza el gobierno argentino con maquetas hápticas en sus museos, incluyendo a Ancona (Italia) donde existe el Museo Tàttile Statale Omèro dedicado exclusivamente a la exhibición de maquetas, esculturas y relieves de la historia del arte.

La impresión 3D nos abre un nuevo mundo de posibilidades que comienza aplicando la tecnología de virtualización $3 D$ al patrimonio. Ambas tecnologías tienen aun más aplicación cuando las enfocamos a la accesibilidad al patrimonio, suponiendo toda una revolución para el acceso visual y háptico, muy especialmente en el caso de objetos de tamaño muy pequeño o muy grande, al permitir la impresión 3D los cambios de escala para aumentar 0 reducir las piezas (Marqués, 2018).

En Puebla (México), se han elaborado maquetas monumentales, como la realizada en bronce del primer cuadro de la ciudad, localizada en la plaza de la Constitución (Zócalo), para posteriormente ser reubicada en el portal Hidalgo, la cual nunca ha tenido una función háptica por su instalación, ya sea por estar colocada en el piso o protegida con algún barandal. 
Estos elementos táctiles ayudan a la identificación de dimensiones, volumetrías y a dar una noción de la espacialidad, y a la difusión del lenguaje arquitectónico, por los detalles poco promovidos; dando al público la oportunidad de poder conocer más detalladamente además de fomentar de la curiosidad para niños y adultos.

\section{Antecedentes}

En México en general, se carece de una cultura sobre accesibilidad lo cual se refleja en una mínima cantidad de proyectos en los que se pueda considerar como un factor importante para este tema. En la línea de investigación Espacio Público, Participación Ciudadana y Centro Histórico, fomentada por la facultad de Arquitectura de la BUAP, se han trabajado diversas iniciativas, tales como el proyecto denominado "Por barrios accesibles y participativos" a través del programa de investigación "Verano Científico CONACYT", en donde se han analizado recintos religiosos en barrios antiguos de la localidad; realizando recorridos a nivel urbano y arquitectónico con sillas de ruedas como dispositivo técnico de movilidad para diagnosticar y establecer itinerarios de acceso a los templos y atrios. Lugares en los cuales se ha involucrado a los "custodios" y propuesto esquemas de accesibilidad; por lo que es necesario establecer nuevas iniciativas para otro tipo de problemáticas

4 Consejo Nacional de Ciencia y Tecnología, México. 5 Guardias de seguridad a las que se enfrentan las personas con alguna discapacidad.

Las dificultad existentes para encontrar proyectos enfocados a las personas ciegas en espacios patrimoniales periféricos, es la razón por la cual en este proyecto de investigación se plantearon estrategias considerando la elaboración de maquetas tridimensionales (hápticas) que contribuyen a la identificación y acercamiento de estas personas con el patrimonio cultural de los templos católicos localizados en el centro histórico de la ciudad de Puebla de Zaragoza (Puebla), con una selección de barrios antiguos de la localidad, como el barrio de San Antonio, El Refugio y Santa Anita.

Estas razones han direcionado esta investigación a la elaboración de un proyecto de accesibilidad al patrimonio cultural dirigido a las personas débiles visuales y ciegos en el centro histórico de la ciudad de Puebla a través de la elaboración de prototipos, maquetas hápticas, planos en relieve y detalles artísticos y arquitectónicos. Además, se planteó involucrar a asociaciones de ciegos y débiles visuales en el diseño y elaboración de las pruebas de los prototipos de las maquetas tridimensionales hápticas de los templos. Se realizó la vinculación entre tiflotécnica (adaptación de los usos y avances técnicos a su utilización por ciegos) y la identificación de elementos simbólicos que definen los detalles y los volúmenes más representativos de los inmuebles, incluyendo la experimentación con diferentes materiales sujetos a la prueba de percepción táctil. 
ACCESIBILIDAD AL PATRIMONIO CULTURAL PARA DÉBILES VISUALES Y CIEGOS EN EL CENTRO HISTÓRICO DE PUEBLA UNA EXPERIENCIA TÁCTIL

Para todo lo anterior, se hizo la selección de un templo de los propuestos inicialmente para realizar un primer modelo volumétrico con la intención de fomentar nuevas alternativas de difusión y en futuras propuestas se puedan gestionar apoyos para multiplicar estos elementos en otros espacios. Dando inicio a las siguientes cinco etapas del proyecto, dos (2) introductorias (Figura 1 y 2) y tres (3) de desarrollo:

1. En esta primera etapa metodológica, el equipo se involucró con el Instituto Educativo para Personas con Ceguera y Debilidad Visual Investigación y Docencia en Educación Especial A. C.

2. Para posteriormente, en la segunda fase, capacitar al grupo en clases de movilidad con bastón y aprendizaje del sistema braille de lectura para ciegos.

3. En una tercera etapa se elaboraron materiales interpretativos de obras pictóricas en alto relieve para la percepción táctil de débiles visuales, incorporando algunas texturas para la interpretación de dibujos y pinturas. Haciendo la evaluación respectiva de que tan explícito puede ser un dibujo con esta técnica.

4. La cuarta etapa, la más larga, fue la elaboración de la maqueta táctil en donde no solo implicó elaborar la maqueta sino saber que programas de cómputo utilizar y plantear etapas de impresión en tres dimensiones y de interpretación de los elementos formales simbólicos de los templos para una lectura táctil.

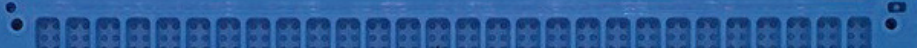

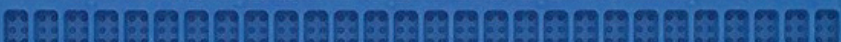

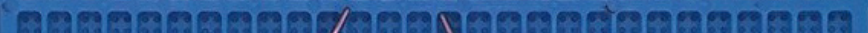
(c) เมะเ

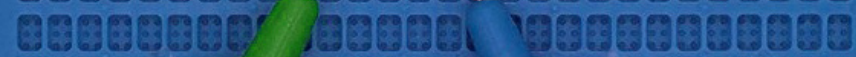

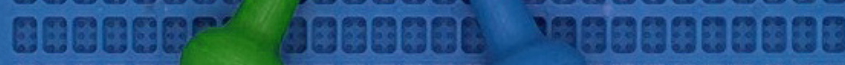
ตำเ 1

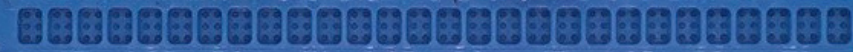

$$
\begin{aligned}
& \begin{array}{llllllllllll}
\text { a } & b & c & d & e & f & g & h & i & j & k
\end{array}
\end{aligned}
$$

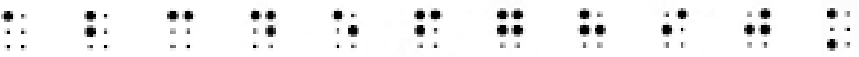

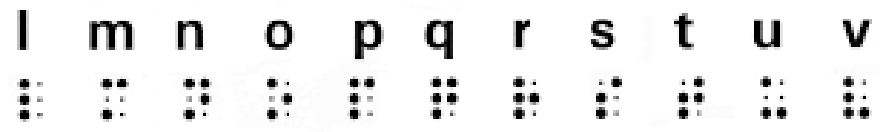

Figura 1. "Pauta” Plantilla Braile.

Fuente: Autor principal.

Figura 2. Foto de primeras pruebas, clases en Braille.

Fuente: Autor principal

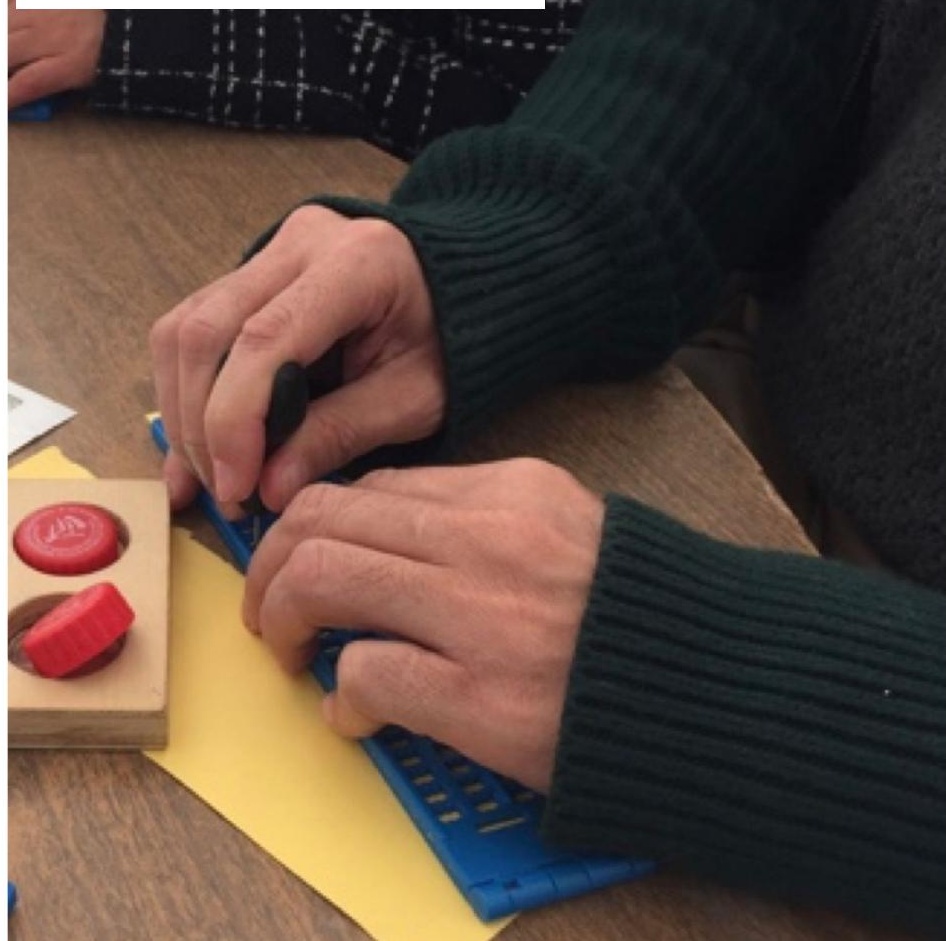


5. La quinta etapa fue la evaluación de la maqueta por parte de las personas débiles visuales y ciegas.

La maqueta física se estableció como un potente método de comunicación en la descripción, exploración y la vinculación de la arquitectura. De hecho, la maqueta lleva más de quinientos años siendo un importante método de comunicación en la compresión de la arquitectura. Aunque el aumento y la evolución de las nuevas tecnologías han permitido que el diseño asistido por ordenador (CAD) se haya convertido en una importante herramienta de diseño arquitectónico, la utilización de maquetas físicas sigue siendo un aspecto fundamental en la educación en esta disciplina y en muchos estudios de todo el mundo (Dunn, 2010, p. 18).

\section{Desarrollo}

\section{Tercera etapa (prueba táctil)}

A través de diferentes técnicas, se realizaron dibujos en alto relieve con pintura inflable sobre un material rígido, con la intención de que se identificaran formas y texturas, para poder explicar a las personas ciegas y débiles visuales la técnica utilizada, así como los antecedentes de la pintura que se representó completa a una escala menor. Por partes, se destacaron algunos de los personajes de la obras escogidas (Pablo Picasso), tomando como referencia básica lo trabajos difundidos en el taller Explora Guernica del museo Centro de Arte Museo Reina Sofía (Figura 3 y 4).

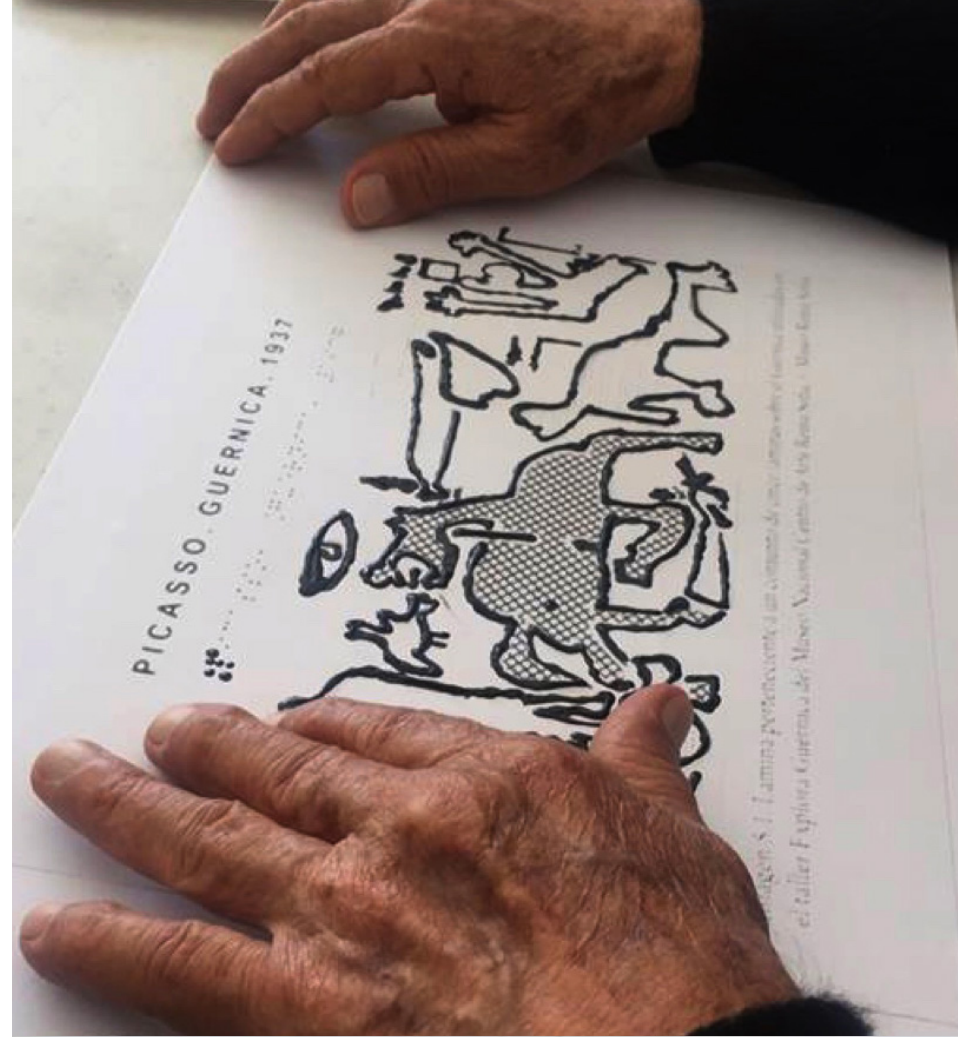

Figura 3. Pruebas táctiles de dibujos en alto relieve.

Fuente: Autor principal.

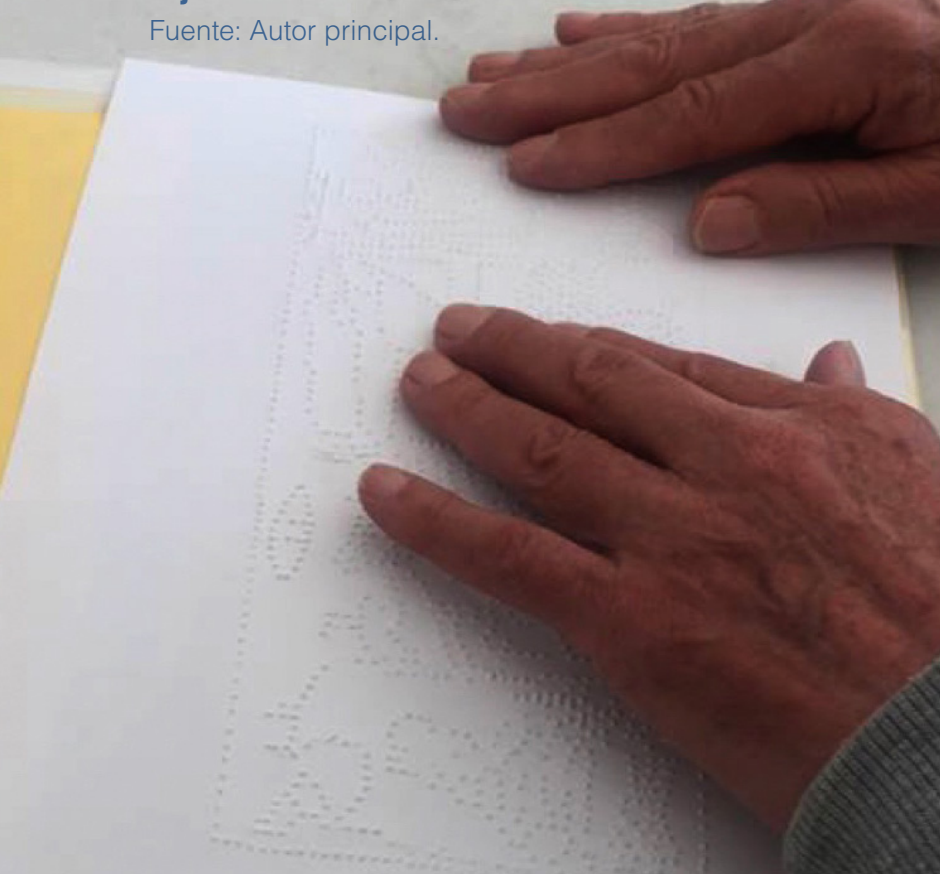

Figura 4. Pruebas táctiles de dibujos en puntuación braile. 
En este primer experimento, fue muy importante para las personas que se les explicara la obra guiando sus manos sobre la silueta de los elementos, que posteriormente identificaron por las diferentes texturas.

Los participantes del grupo encuestado destacaron la importancia de la previa explicación oral para poder entender los dibujos, por la difcultad para identificarlos por sí solos. Destacaron los dibujos con una mejor evaluación por las personas ciegas y débiles visuales los que poseían trazos más sencillos con mayores protuberancias perceptibles.

\section{Cuarta etapa}

(Maqueta táctil)

Para el desarrollo de la presente etapa del trabajo de investigación, se escoge únicamente (Figura 7) un templo de los tres (3) preseleccionados anteriormente debido a que los procedimientos planteados para la impresión estuvieron sujetos a los tiempos del laboratorio de fabricación digital FAB $L A B$ de Puebla. Las dimensiones de la maqueta se replantearon a una escala de 1:100 con la intención de que el objeto fuera abarcable a una distancia de 90 centímetros (brazos abiertos). Por lo anterior, y sumando el costo del material, se imposibilitó realizar los tres elementos hápticos planteados en un inicio, por lo que este proyecto se debe considerar a largo plazo (3 años).
Figura 5. Dibujo a mano alzada.

Fuente: Autor principal.

Figura 6. Dibujo en puntuación braile.

Fuente: Autor principal.
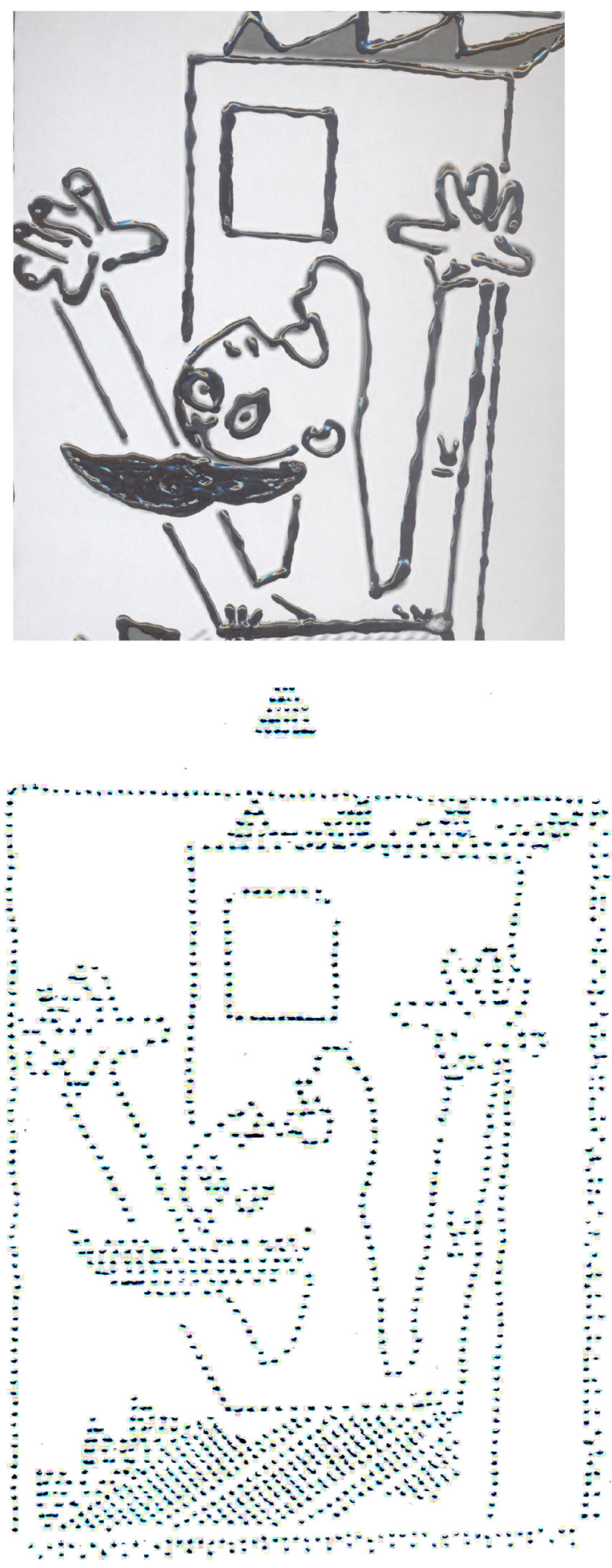
Las maquetas, reproducciones y replicas táctiles deben corresponder a piezas lo más representativas posible de la exposición o el museo, para que transmitan la mayor cantidad posible de valores, mensajes principales e informaciones culturales coherentes con el contenido, el discurso museográfico y los fines principales de la colección, y no caer en el error de adaptar o utilizar otras secundarias o menos interesantes, aunque por sus características resulten más sencillas, fáciles o baratas de reproducir. Siempre se debe en cualquier caso primar la eficacia en la transmisión de los contenidos a otros factores (Espinoza y Bonmatí, 2013, p. 111).

Las especificaciones de la maqueta elaborada son:
- Área de maqueta (90 cm. x 60 cm).

- Altura (20 centímetros)

- Color azul (sky blue)

- Material (COLORFABB PLA/PHA polihidroxialcarbonato, diámetro 2.85 mm.

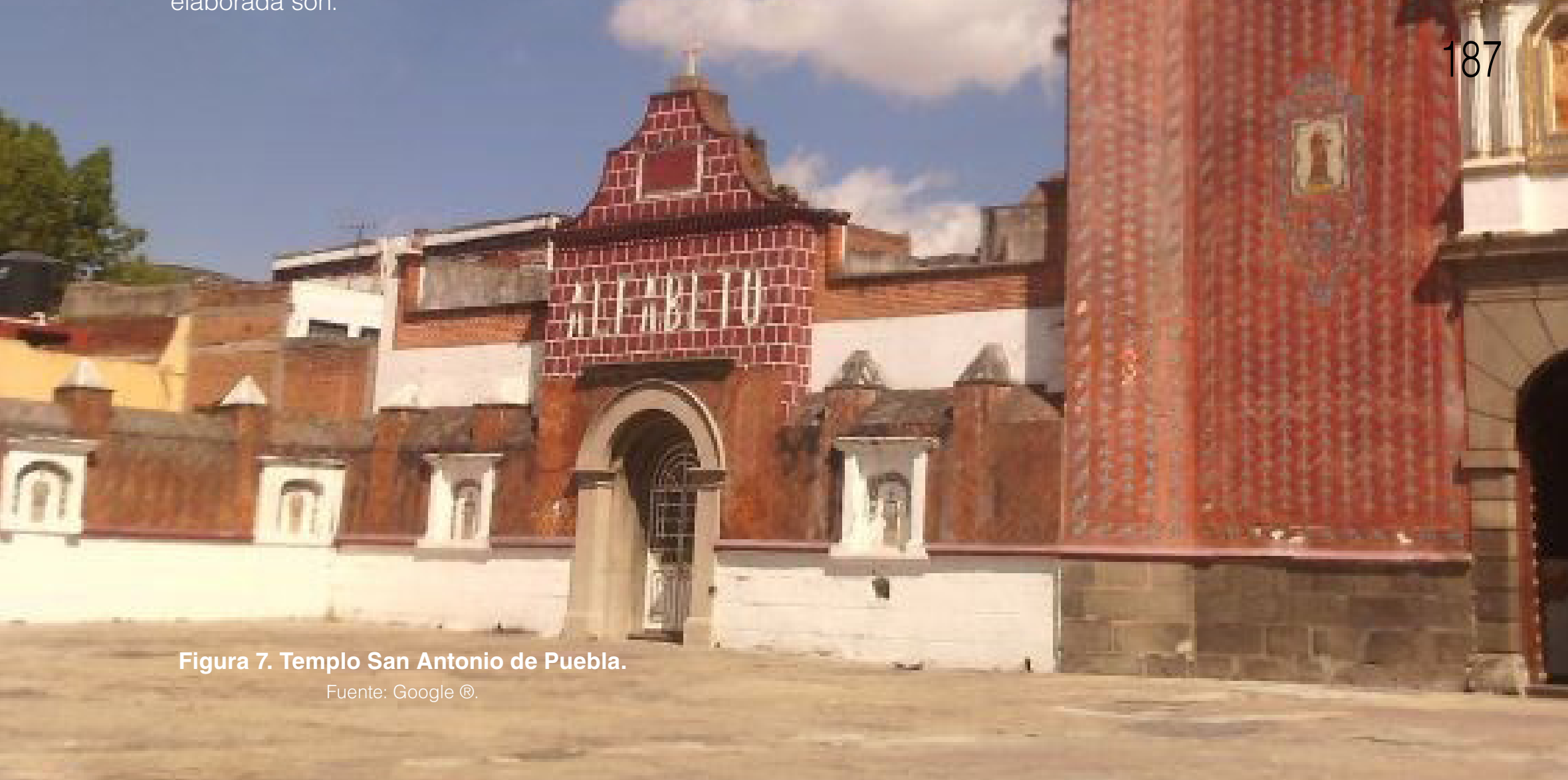




\section{SubEtapas}

a. Reconocimiento del inmueble Templo de San Antonio. Levantamientos arquitectónicos de la planta arquitectónica y alzados de la misma (figura 8 y 9$)$. b. Elaboración de modelos 3D en diferentes programas (Auto Cad y Rhinoceros) para visualizar el volumen en color monocromático con el objetivo de resaltar elementos de importancia: cúpula central, bóvedas, pretiles, espadañas, pináculos, entrada principal y fachada interna del templo.

Figura 8. Vista en perspectiva frontal del modelo $3 \mathrm{~d}$ realizado en RHINOCEROS.

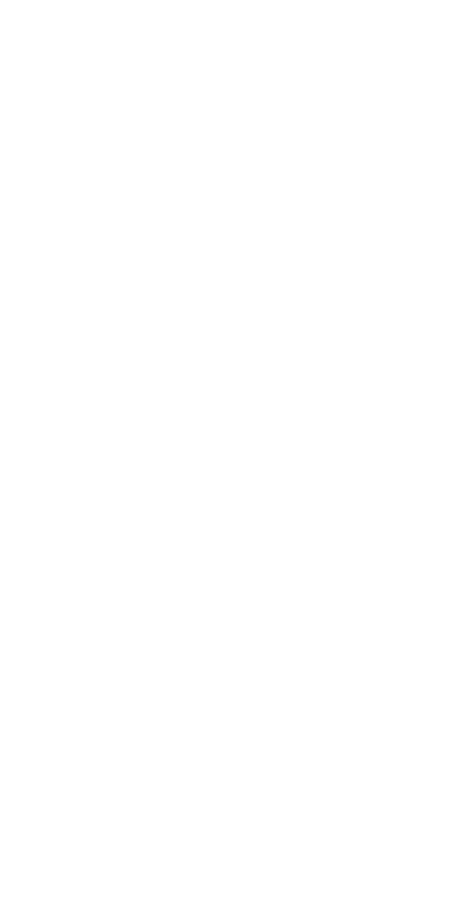

$\square$ fin $\square$ Cerca $\square$ Punto $\square$ Med $\square$ Cen $\square$ it $\square$ Perp $\square \operatorname{Tan} \square$ Cuad $\square$ Nodo $\square$ Veticice $\square$ Proyectar $\square$ Desactivar

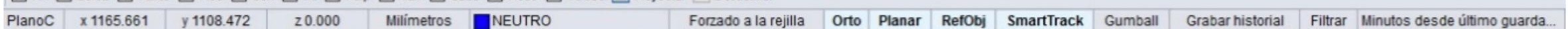


Dentro de las recomendaciones del FAB $L A B$, se indicó dar prioridad a elementos con más detalles que aquellos de cara lisa principalmente por el proceso que efectúa la impresora modelo Ultimaker.
La impresión por piezas se inició con la zona del atrio del templo, los muros perimetrales y el acceso, los cuales incluyeron los detalles de los nichos en el muro interno y destacando el muro frontal (entrada al salón Alfabeto).

Figura 10. Proceso de impresión de piezas realizado en el FAB LAB.
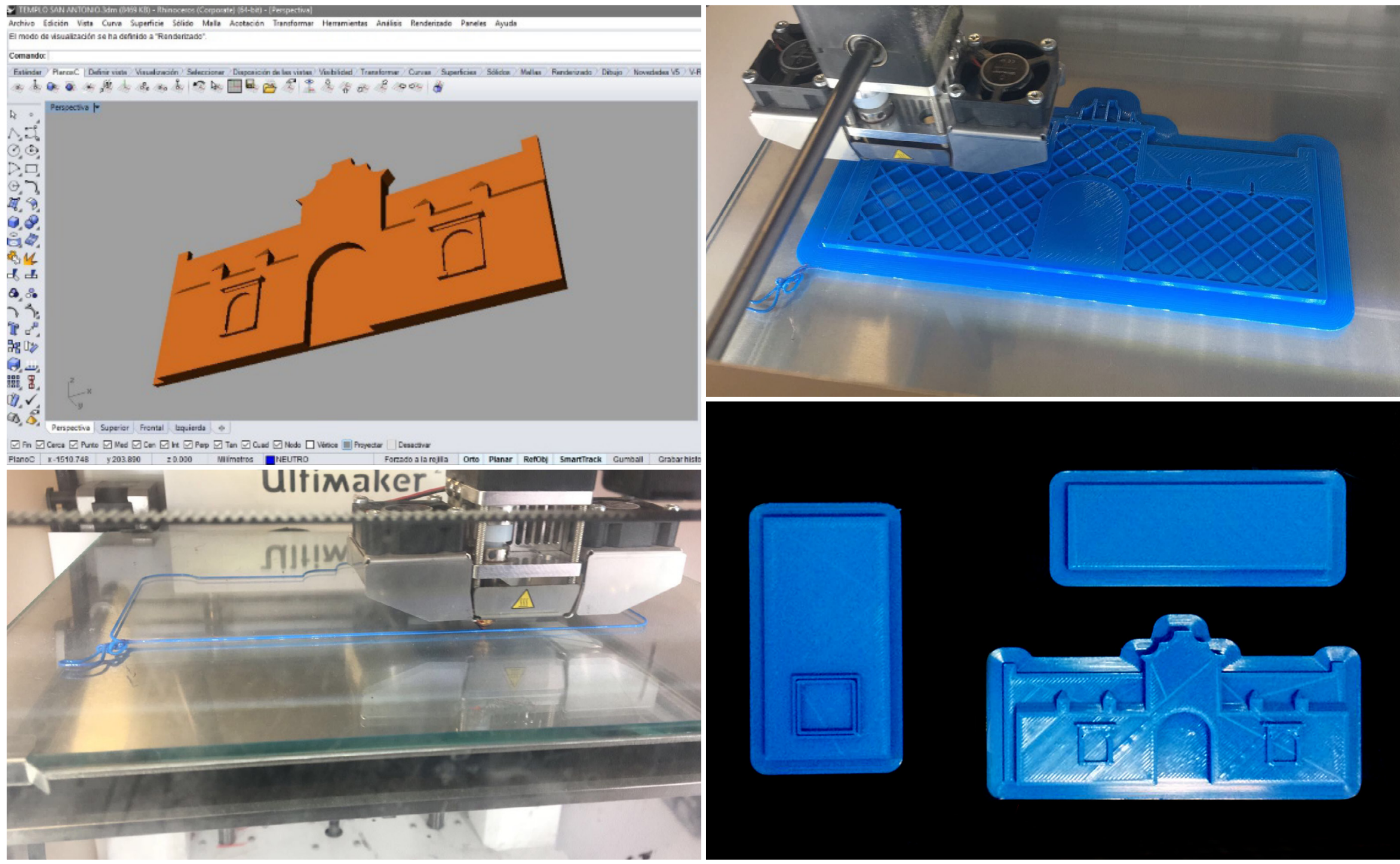


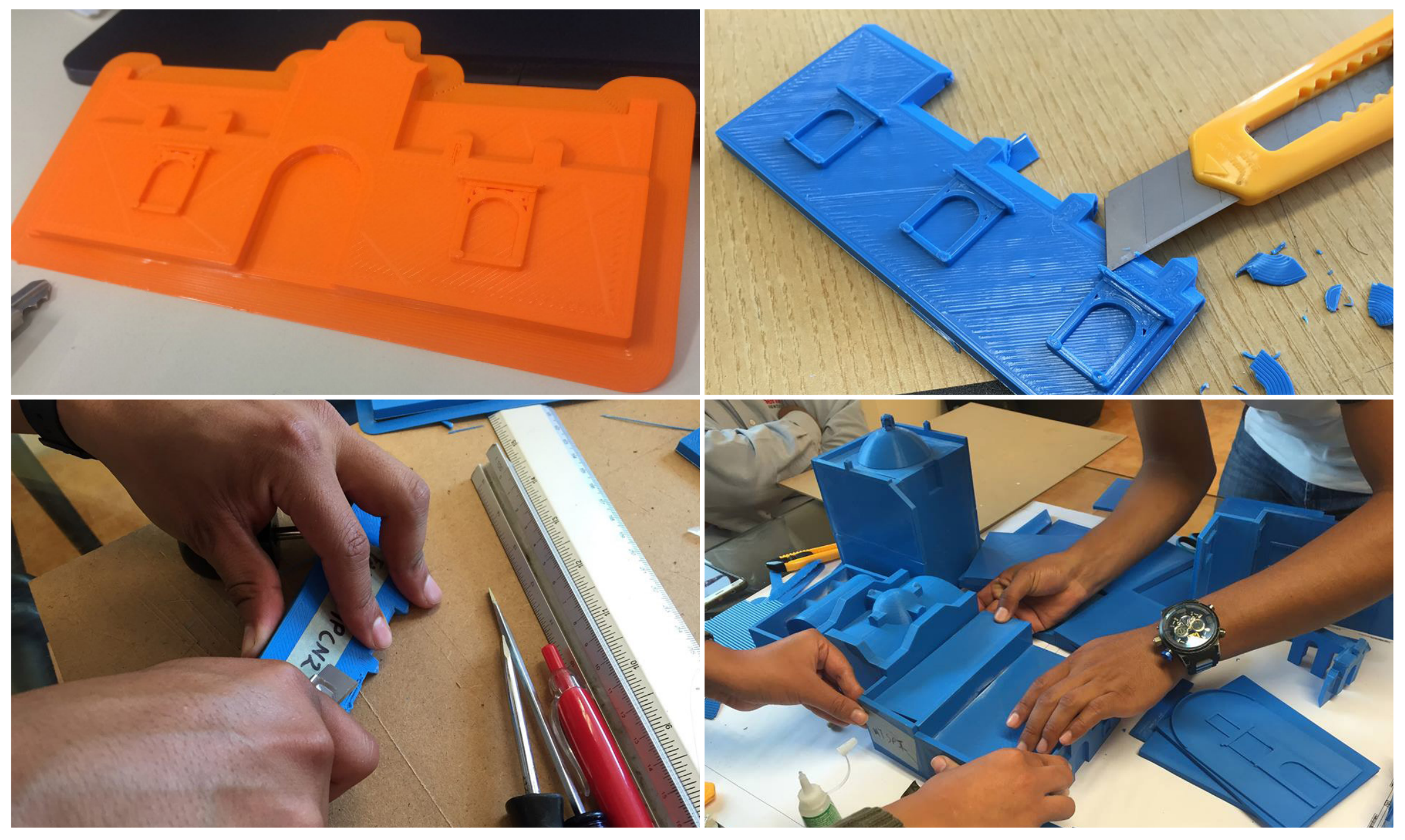

Figura 11. Proceso de lijado y pegado de piezas.

Fuente: Autores.

El proceso de impresión conlleva la separación de algunos elementos, por sí estos superaban las medidas máximas de la máquina de impresión, continuando con la exportación de cada una de las piezas al formato ".DWG", con la intensión de tener un archivo manipulable para su exportación a la extensión "STL", para enviar a la impresora 3D. e. El ensamble de la maqueta se realizó con el desprendimiento del material sobrante y el lijado de las piezas en donde se unieron con pegamento instantáneo industrial de alta resistencia transparente. Este trabajo se realizó por módulos para facilitar la transportación de la maqueta (figura 11). 
La maqueta esta constituida por más de 50 piezas que consumieron 7 rollos de material COLORFABB, con las siguientes especificaciones:

- PLA/PHA 2.85 MM SKY BLUE PLA, PHA (polihidroxialcarbonato), es un bio-poliester 100\% biodegradable.

- Tolerancia de diámetro uniforme +- $0.05 \mathrm{~mm}$

- Temperatura de impresión $195-220^{\circ} \mathrm{C}$

- Peso 750 gramos por rollo.

f. Se desarrollaron impresiones de algunas piezas con tres colores para realizar pruebas correspondientes al color más visible para las personas débiles visuales: azul (sky blue), verde (fluorescent green) y naranja (dutch orange).

\section{Resultados}

Para la evaluación de la maqueta en 3D se pudo trabajar con personas débiles visuales y ciegos con el objetivo de que pudieran identificar los volúmenes de un templo antiguo, así como distinguir por el tacto diferentes elementos arquitectónicos de un edificio.

La maqueta fue evaluada por dos grupos de personas con ceguera y debilidad visual. El primero estaba conformado por tres integrantes del Instituto Educativo para Personas con Ceguera y Debilidad Visual. Investigación y Docencia en Educación Especial A.C. (IECEDEVI) con sede en Cholula (Puebla). Tres adultos mayores que perdieron la vista a causa de enfermedades como la diabetes o derivado de

Tabla 1

Relación de personas que evaluaron la maqueta táctil.

\begin{tabular}{|c|c|c|c|c|c|}
\hline Nombre & $\begin{array}{l}\text { Edad } \\
\text { (años) }\end{array}$ & Ocupación & $\begin{array}{c}\text { Tipo de } \\
\text { discapacidad } \\
\text { visual }\end{array}$ & $\begin{array}{c}\text { Causa de ceguera o } \\
\text { debilidad visual }\end{array}$ & $\begin{array}{c}\text { Tiempo con } \\
\text { ceguera o debilidad } \\
\text { visual (años) }\end{array}$ \\
\hline $\begin{array}{l}\text { Virgilio } \\
\text { Hombre } 1\end{array}$ & 70 & Profesor & Ceguera & $\begin{array}{l}\text { Derivada de un } \\
\text { traumatismo a causa de } \\
\text { una explosión en el rostro. }\end{array}$ & 45 \\
\hline $\begin{array}{l}\text { Patricia } \\
\text { Mujer } 1\end{array}$ & 63 & $\begin{array}{l}\text { Profesora } \\
\text { jubilada }\end{array}$ & Ceguera & Glaucoma & 10 \\
\hline $\begin{array}{l}\text { Paulino } \\
\text { Hombre } 2\end{array}$ & 57 & $\begin{array}{l}\text { Mecánico } \\
\text { automotriz }\end{array}$ & Ceguera & Glaucoma & 4 \\
\hline $\begin{array}{l}\text { Juan } \\
\text { Hombre } 3\end{array}$ & 45 & $\begin{array}{l}\text { Ingeniero } \\
\text { en } \\
\text { sistemas }\end{array}$ & $\begin{array}{l}\text { Debilidad } \\
\text { visual }\end{array}$ & Retinitis Pigmentosa & 20 \\
\hline $\begin{array}{l}\text { Ángeles } \\
\text { Mujer } 2\end{array}$ & 42 & Odontóloga & $\begin{array}{l}\text { Debilidad } \\
\text { visual }\end{array}$ & Retinitis Pigmentosa & 20 \\
\hline
\end{tabular}

Fuente: Autores. 
accidentes laborales. De ellos, uno ha sido ciego más de la mitad de su vida, mientras que los otros dos perdieron la vista hace menos de diez años.

En el caso del segundo grupo, se trata de dos adultos con Retinitis Pigmentosa y que se percataron de su condición al concluir sus estudios universitarios (Tabla 1).

La primera parte de la evaluación consistió en permitir a las personas tocar la maqueta mientras uno de los integrantes del equipo cuenta la historia del templo y describe los espacios y elementos arquitectónicos del edificio.

Al concluir, se realiza un cuestionario con diez preguntas que contestaron en el primer grupo Patricia (Mujer 1), Virgilio (Hombre 1) y Paulino (Hombre 2) en las instalaciones de IECEDEVI. Mujer 2 y Hombre 3, ambos con Retinitis Pigmentosa.

Primera pregunta: Después de haber escuchado la explicación y la historia del templo, así como de haber tocado la maqueta, ¿tiene una mejor idea de cómo es un templo antiguo? La mayor parte de los participantes apoyaron la importancia de una explicación del templo para poder identificar las principales partes del mismo, así como en otros casos era un templo conocido para las personas o ya había sido visitado con anterioridad.

Segunda pregunta: ¿Pudo identificar las diferentes áreas del templo según lo que le indicó el instructor? El 100\% de los encuestados respondió que los elementos como cúpulas, espadañas y las referencias que conocían era un factor determinante para entender el espacio; en algunos casos los nombres eran muy técnicos, razón por la cual no los recordaban con precisión, haciendo destacable la uitlidad que tiene la maqueta para poder conocer con mayor detalle el templo, ya que por lo general solo se entra a lugares específicos.

Tercera pregunta: ¿La escala (o tamaño) de la maqueta ayuda a entender el espacio? La mayoría dijo que sí, en un $80 \%$, destacando entre las ventajas la facilidad para tocar con las manos los detalles al no ser muy pequeños, mientras el 20\% opinó que debía ser más grande.

Cuarta pregunta: ¿Pudo percibir con el tacto las diferentes texturas de la maqueta? El $100 \%$ de las personas pudieron percibir las texturas, y mencionaron que eran agradables al tacto, pero que había algunos bordes un tanto filosos

Quinta pregunta: ¿Considera que se requiere una mayor explicación del instructor para entender el espacio o sólo con tocar la maqueta es suficiente? El $100 \%$ comentó que es necesaria una explicación, y algunos prefieren que mientras van tocando se escuche la explicación y se ahonde un poco más; inclusive una sugerencia fue la posible incorporación de audio.

Sexta pregunta: ¿Es necesario contar con una planta arquitectónica (un plano) al lado de la maqueta? El 40\% opinó que sería necesario, pero que tenga textos y abreviaturas, tanto en Braille y en letras con relieve. Por temas de ubicación y del recorrido que se pudiera hacer en el interior. 
Séptima pregunta: Si sabe leer en Braille, ¿considera que cada espacio debe contar con un texto en Braille para identificarlo mejor? El 60\% dijo que no era necesario colocar simbología, ya que estaban en el proceso de aprendizaje, el resto sugería su incorporación.

Octava pregunta: Si usted es débil visual, ¿cree que el color de la maqueta es el adecuado? En temas de color sólo las personas con debilidad visual sugerían el contraste de colores, y que el azul era un buen tono porque no lastima a la vista.

Novena pregunta: ¿Sería importante conocer el espacio en el sitio real? El $100 \%$ sugería que era importante que posterior a la explicación se hiciera una visita al templo como ejercicio.
Décima pregunta: ¿Algún comentario o sugerencia para el mejor funcionamiento de la maqueta? ¿Alguna recomendación? Dentro de las recomendaciones realizadas se sugiere que para la maqueta cumplir una función didáctica, de difusión y también de recreación, es necesario contar con un reglamento para que la gente no la destruya y considerar también el tema del mantenimiento.

Con relación a la calidad de la maqueta, se mencionó lo desagradable de las partes filosas y las zonas rasposas desagradables; además de por seguridad evitar que las personas se puedan cortar con alguna rebaba o filo. Como comentario, también es necesario considerar que los colores fueran contrastantes.

\section{4}

Figura 12. Pruebas táctiles.

Fuente: Autor principal.
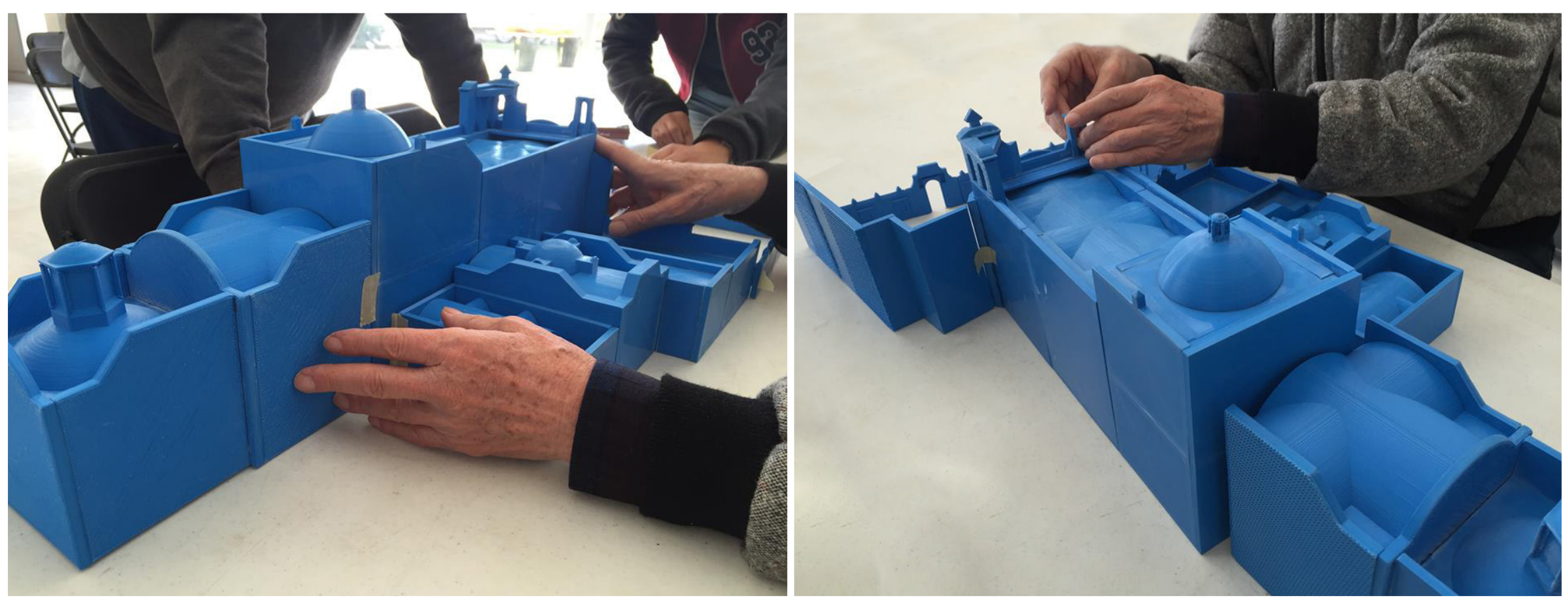


\section{REFERENCIAS}

Ayuntamiento de Puebla. (2013). Manual ciudadano para conocer la discapacidad. Puebla: Ayuntamiento de Puebla.

Ayuntamiento de Puebla. (2011). Manual Técnico de Accesibilidad aplicable a construcciones en el municipio de Puebla. Puebla: Ayuntamiento de Puebla.

Blanco, L. (1998). Un práctico sistema para la elaboración de mapas táctiles. Integración (28), 25-28.

Buenfil, T. (2007). Diseño Universal / Aspectos Teóricos. En, Memorias del Coloquio de Diseño para Todos, Entornos, Espacios y Objetos (43-44). UNAM.

Centro de Investigación, Desarrollo y Aplicación Tiflotécnica (ONCE - CIDAT). (2015). Catálogo Tiflotécnico 2015. Madrid: ONCE- CIDAT.

Consuegra, B. (1998). Maquetas accesibles a las personas con discapacidad visual. Integración (28), 16-20.

Corporación Ciudad Accesible. (2010). Manual de Accesibilidad Universal. Santiago: Ciudad Accesible.

De La Torre, C. (2015). Arquitectura con valor patrimonial para todos. Caso Catedral Basilica de la ciudad de Puebla. [Tesis]. Puebla: BUAP.
De La Torre, C., Hernández, A. y Santamaría, C. (2015). Anteproyecto ejecutivo de intervención para la accesibilidad de personas con discapacidad motriz a la Catedral Metropolitana de la ciudad de Puebla. Puebla: Re-Genera Espacio.

Espinosa, A. y Bonmatí, C. (Ed.). (2013). Manual de accesibilidad e inclusión en museos y lugares del patrimonio cultural y natural. Gijón: Trea.

García, D. (2014). Centros Históricos Accesibles. En, Segundo Seminario Internacional de Estudios sobre Centros Históricos. Prácticas y políticas de conservación. México D. F.: CyAD UAM Xochimilco.

Gómez, Á. (2015). La accesibilidad para personas ciegas y con deficiencia visual al patrimonio cultural y natural. Her\&Mus 16, 7(I), 47-54. Recuperado de http:// www.raco.cat/index.php/Hermus/ article/download/313253/403378

Hernández, A., Abarca, K., Aguilar, G., Cabrea, A., López, P., López, K., Mejía, C., Rodríguez, C. y Torres, R. (2015). Barrios Accesibles: Espacio Público y Templos San Antonio, El Refugio y Santa Anita. Centro Histórico de Puebla. Por, Barrios Accesibles y Participativos. Verano de Investigación 2015. Puebla: BUAP.

Institut Municipal de Persones amb Discapacitat. (2009). 30 anys treballant amb les persones amb discapacitat. Una ciutat per a tothom: Barcelona. Barcelona: Ajuntament de Barcelona. 
Marqués, N. (2018). La impresión 3D para sentir el patrimonio. Recuperado de http://nestormarques.com/impresion3d-para-sentir-el-patrimonio/

Moyssen, M. (2013). Diseño Industrial Incluyente. En, O. Salinas, Diseño Industrial. Formación, propósito y acciones (129-139). México, D.F.: UNAM.

Organización Nacional de Ciegos Españoles. (2006). Características de la rotulación para personas con discapacidad visual. Madrid: ONCE.

Regatos, R., Portolés, J. y Mera, J. (2003). Carencias y Oportunidades de la I+D para la Innovación en Accesibilidad Urbanística y en la Edificación. En, C. Minusválidos, Libro Blanco I+D+l al servicio de las Personas con Discapacidad y las Personas Mayores. (267-306). Valencia: CERMI.
Revilla, F. (2007). Diccionario de iconografía y simbología. Madrid: Cátedra.

Secretaría de Desarrollo Urbano y Vivienda. (2007). Manual Técnico de Accesibilidad. México, D.F.: Gobierno del Distrito Federal. Recuperado de http://www.libreacceso. org/downloads/Manual\%20de\%20 Accesibilidad\%20SEDUVI.pdf

Tecnoayudas. (2010). Tecnoayudas. Recuperado de http://www.tecnoayudas.com/index.php 